\title{
EN LOS MÁRGENES \\ DE LA LEGITIMIDAD. EXCLUSIÓN Y CIUDADANÍA
}

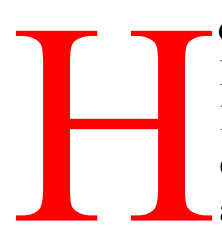

oy no es difícil encontrar argumentos que permiten vincular las nociones de Estado y exclusión. Sólo con examinar cualquier informe sobre la situación de los derechos humanos, parece claro, por ejemplo, que la supervivencia de una condición que define ese vínculo, la categoría de ciudadano en contraposición a la condición de extranjero (a fortiori, de refugiado, o apátrida), constituye un argumento decisivo a favor de la inaplazable necesidad de redefinir la soberanía estatal, o, mejor, de superar las condiciones que ésta plantea a aquéllos, a los derechos. No basta con esgrimir la distinción clásica entre derechos del hombre y del ciudadano. Problemas como los derivados de la inmigración hacia la Unión Europea, o las demandas planteadas por las minorías culturales -no digamos si se trata de las minorías nacionales- han contribuido a poner de relieve tales insuficiencias.

Sin embargo, no menos claro se me antoja -y cada vez más- que sostener de un modo simplista la vinculación entre Estado y exclusión propicia efectos perversos, tal y como los que quiero plantear en estas páginas, aprovechando la oportunidad del número monográfico de Doxa dedicado a Elías Díaz. En efecto, son sus tesis, de todos conocidas, las que querría tomar precisamente como punto de partida para tratar de exponer algunos de los riesgos y errores que comporta esa identificación. Por esa razón, a nadie que conozca los trabajos de Elías Díaz ${ }^{1}$ le sorprenderá que se discuta aquí, de nuevo, acerca de la «maldad estatal», los límites de la sociedad civil y del individualismo, así como de las instituciones -el mercado- que son

${ }^{1}$ Por referirme sólo a algunos de los últimos, por ejemplo, De la maldad estatal y la soberanía popular, Debate, Madrid, 1984; La transición a la democracia, Eudema, Madrid, 1987, Ética contra política. Los intelectuales y el poder, Madrid, CEC, 1990. 
su paradigma, al menos en la versión que es hoy canónica, la liberal. Tampoco se llamará a andana porque, recordando otro de sus temas clásicos, la relación entre los intelectuales y la política, trate de echar un cuarto a espadas acerca de la (re)definición de las relaciones entre universalismo e izquierda política, a propósito de las dificultades de conciliación entre «identidad cultural» $\mathrm{y}$ «patriotismo constitucional». Y eso es lo que trato de hacer tomando como pretexto la vinculación entre Estado y exclusión, porque, en definitiva, si tienen sentido este tipo de homenajes -en mi opinión, desde luego- es para continuar discutiendo con el homenajeado. Ciudadanía y mercado de trabajo son los dos ejes sobre los que, de acuerdo con un análisis nada insólito ${ }^{2}$ trataré de enunciar algunas propuestas en torno a ese nexo que parece unir hoy Estado y exclusión.

El problema, como advierte $\mathrm{Veca}^{3}$, reside precisamente en la paradoja de que el mito moderno de la identidad, al mismo tiempo que crea cohesión, funciona también como mecanismo de exclusión. Ese mito, además, es el que subyace a la moderna noción de ciudadanía (y a su vínculo con la nacionalidad, tal y como la heredamos de la Revolución Francesa, que está en el origen de las modernas concepciones del Estado, del Derecho, de la ciudadanía). En otras palabras, lo que hoy se insiste en denunciar, como lo hacen por ejemplo Barcellona ${ }^{4}$ (siguiendo los pasos de Habermas) o Ferrajoli ${ }^{5}$, es que fenómenos como el nuevo (des)orden económico y político internacional, junto al impacto de la transformación de la inmigración procedente del tercer mundo y del Este de Europa, o síntomas como el incremento del racismo y la xenofobia, afectan decisivamente al núcleo del vínculo social mismo, al fracturar algunos de los supuestos sobre los que se edificaba un pretendido universalismo, esto es, la uniformidad que el proyecto del formalismo jurídico y del liberalismo político y económico habían tratado de poner en pie. En este sentido, lo que procede es plantear la pregunta acerca de si la existencia misma de la comunidad constituye el factor primordial de exclusión, por exigencia ontológica, digámoslo así, o si es el carácter

${ }^{2}$ Me limitaré a recordar uno de los más conocidos, el de Balibar, que califica al paro y la exclusión de ciudadanía como las dos grandes modalidades de exclusión: «Inegalités, fractionnement social, exclusion» (en VV.AA., Affichard-De Foucault, eds.), Justice social et inegalités, París, Esprit, 1992, pág. 157 y ss.

3 S. Veca, Cittadinanza. Riflessioni filosofiche sull'idea di emancipazione, Milano, Feltrinelli, 1990; en el mismo sentido, los trabajos de Leca, como Leca, J «Nazionalitá e cittadinanza nell'Europa delle immigrazioni», en VV.AA., Italia, Europa e nuove immigrazioni, Torino, Ed. Fondaz. Agnelli, 1990. Trotta, 1993.

${ }^{4}$ Cfr. p. ej. su Postmodernidad y comunidad. El regreso de la vinculación social, Madrid,

${ }^{5}$ Cfr. su Diritto e Ragione. Per una teoria generale del garantismo penale, Bologna, 1989, en especial la última parte del libro. 
político de ciertas comunidades (por excelencia, el Estado) o bien otro tipo de factores lo que potencia hoy la exclusión.

Como se ha recordado con no poca frecuencia, lo primero que nos sugiere la tesis de la exclusión en relación con el Estado es que se puede predicar de la historia de los Estados el lema «... en el principio fue la exclusión». La aparición de esas formas de organización política se basa en la contraposición entre nacionales y extranjeros, y en la exclusión de éstos. Como recuerda Sami-Naïr, si la soberanía es el mecanismo de inclusión/exclusión que entendemos justificado, el Estado tendría como trabajo principal gestionar esa inclusión/exclusión legítima y la política sería el proceso de formación de la soberanía. Por tanto, los excluidos de la política, los que están privados de la participación en la formación de la soberanía, quienes están fuera del Estado, son los radicalmente excluidos, los sujetos de lo que en otro lugar he llamado la exclusión «natural». El Estado, en feliz expresión del politólogo francés, tiene «la llave de la diferencia», es quien instituye a través del Derecho la noción misma de extranjero, como la de minoría, esto es, las modalidades de exclusión. Por eso puede decir Facchi que «el trabajo cotidiano del juez consiste en definir lo semejante y lo diferente... problema que se torna más complicado cuando se trata de deslindar no ya comportamientos diferentes, sino comportamientos de individuos que pertenecen a categorías sociales $<$ diferentes $>\gg{ }^{6}$.

Incluso la democracia, no hace falta insistir en ello, tiene en su origen el mismo principio, que es clave de su prosperidad económica: baste recordar el modelo griego clásico. Como también suele recordarse, es precisamente la fuerza emancipadora de los derechos la que introduce en la democracia moderna un rasgo expansivo, una línea que rompe progresivamente los círculos de cierre, acogiendo cada vez a más amplias sectores y categorías de personas como sujetos, aunque, como sabemos bien hoy ${ }^{7}$, todavía quedan ámbitos de exclusión. Aún podríamos decir más, especialmente los extranjeros representan hoy -en tanto que exclusión «natural»- un vestigio histórico de la evolución de las nociones de Estado y ciudadanía: el camino recorrido por la burguesía primero, por los asalariados después, aún no ha sido transitado por ellos, que continúan en una situación más parecida a la de súbditos -siervos- que a la de ciudadanos (soporte económico real, en cuanto mano de obra, y a la par sin derechos; poco a poco, lograrán los derechos civiles y sociales; no los

111.

${ }^{6}$ Facchi, A., «L'escissione: un problema giudiziario», Sociologia del diritto XIX/1992, pág.

${ }^{7}$ He tratado de argumentarlo en El desafio de las fronteras, Madrid, Temas de Hoy, 1994. 
políticos). La historia es, en gran medida, el proceso de superación de los ámbitos de exclusión, y eso se ve muy claro con los derechos humanos.

Quizá, pese a todo, sea necesario advertir que no pretendo sumarme hoy a las cohortes que aclaman el nuevo carro del vencedor, con sus entusiastas proclamas de «imenos Estado!» como axioma sagrado y principio indiscutible del «nuevo» orden mundial. En ese sentido, no cabe satanizar el Estado frente a otras formas de comunidad política. No sólo porque se pueda sostener, como haré, que el «exceso» de Estado no es el más peligroso de los males: al fin y al cabo, volvamos al guiño hegeliano, el Estado es el espacio de los derechos, de la libertad (o sea, es la condición de ciudadano lo que nos permite, al menos, plantear los conflictos), sino porque las otras modalidades no ofrecen una superación de la cuestión. No parece, por ejemplo, que los imperios funcionen como factores reductivos de la exclusión. Desde luego, en todo caso no creo que lo sean menos que el Estado nacional. Que los imperios -el último ejemplo, los coloniales- se basan en la exclusión institucionalizada, que se asientan en la clara distinción entre el auténtico ciudadano (el miembro de pleno derecho, que es ciudadano de la metrópolis) y los demás es una lección histórica difícilmente refutable.

En todo caso, creo que el punto de partida puede ser una afirmación que quizá resulte provocativa en los tiempos que corren, sobre todo por el inequívoco aroma «comunitarista» que olfatearán enseguida los inefables policías del pensamiento ${ }^{8}$, máxime si se revela de inmediato su autor, M. Walzer. Pues bien, se trata de reconocer que la condición de ciudadanía, o, si se prefiere, de pertenencia (de «membrecía», traducen algunos) a un grupo, a una comunidad (al Estado, no en balde «sociedad perfecta», que decían los viejos manuales de ciencia política, hegelianos al fin) es un bien, e incluso más, uno de los bienes primarios ${ }^{9}$. En ese sentido, ante el problema

${ }^{8}$ No me refiero a la moderna versión de los inquisidores -afortunadamente, apenas quedanilustrada por Orwell en su 1984, (un libro del que guardo un recuerdo que de nuevo me remite a Elías, porque fue el texto elegido por el tribunal del que él formaba parte para el tercer ejercicio de una oposición en la que participé desde el otro lado; y que salió bien, que es otro motivo para evocarlo), sino a una versión menor, la de tanto académico con vocación frustrada de policía local o guardia de tráfico que sigue creyendo en la necesidad -más o menos analítica: noble coartada- de adscribir inequívocamente los conceptos, análisis y propuestas que formule cada quien a una u otra de las direcciones doctrinales en liza, quizá porque lo decisivo les parezca la identificación rigurosa e inequívoca, más que la contribución a los problemas reales que, ya se sabe, en realidad no importan, porque no se pueden resolver, o al menos no es esa nuestra competencia (una vez más, los parónimos análisis y analgesia).

${ }^{9}$ Esa es la tesis básica de Walzer en su Spheres of Justice. No es casual que el capítulo de ese libro que lleva por título «Membership» fuera elegido para ilustrar su 
que plantea la disyuntiva aparentemente irreconciliable entre universalismo y particularismo, convendría señalar algunas precauciones.

En primer lugar, y frente a los partidarios de un universalismo tan ideal como vaporoso que muchas veces sólo a duras penas logra ocultar su carácter pro domo sua, tal y como subrayara Montaigne ${ }^{10}$ en su ensayo a propósito de las costumbres de los caníbales (es decir, a proposito del primer gran choque cultural, el del nuevo mundo, cuyas diferencias son justamente la coartada para tratar de legitimar la dominación, porque se trataría de costumbres antinaturales, tal y como se subraya en la polémica acerca de los justos títulos ${ }^{11}$ ) cuando escribe que «llamamos barbarie a lo que no entra en nuestros usos», y compara los sacrificios humanos con las tortura de la Inquisición, para concluir irónicamente, tras mostrar no pocas muestras del buen juicio de los «salvajes» frente a lo que tenemos por evidencias, «Buena cosa parece todo esto, pero ¿vamos a tener en consideración a unos individuos que no llevan calzones?». Habrá que recordar que lo único que resulta imprescindible es la distancia crítica con la propia cultura, que permita a la vez, separarse de lo que son prejuicios, sin intentar habitar esa suerte de mundos supralunares, como los calificara I. $\operatorname{Berlin}^{12}$, ajenos al propio lenguaje y al propio universo moral (aunque esto tiene dificultades).

Al mismo tiempo, no por admitir lo anterior tendríamos que dejar de reconocer que pese a esto, o, mejor, precisamente por ello, toda comunidad comporta exclusión, y la forma más perfeccionada de pertenencia a una comunidad (el Estado), exasperaría, pues, esa deficiencia, tal y como lo mostraría la constitución de la dicotomía básica ciudadano-extranjero, $y$, sobre todo, la traducción de la misma en los conceptos schmittianos freund-feind que resumirían toda una visión de la política que tiene entre otros eslabones el de Rousseau ${ }^{13}$,

posición en uno de los más conocidos readings sobre el debate entre comunitarismo y liberalismo. Otro destacado comunitarista, Taylor, se ha ocupado de lo que constituye sin duda la peor cara de este movimiento - la relación entre comunidad y exclusión- en su Multiculturalism (cito por la trad. francesa, Multiculturalisme, Democratie et Difference, París, 1993, Aubier, que incluye los textos del debate con Rawls y Walzer).

${ }^{10}$ Montaigne, Essais, L. I, XXX; cito por la edic. de Ensayos completos, vol. I, págs. 150 ss.

${ }^{11}$ Sobre ello, Pérez Luño, A. E, La polémica del nuevo mundo, Madrid, Trotta, 1992.

${ }^{12}$ Cfr. Conceptos y categorías. Ensayos filosóficos, México, F. C. E., 1992.

${ }^{13}$ Aunque Rousseau ofrezca argumentos para más de una interpretación. A quienes aduzcan el conocido texto del Emilio, «Todo patriota es duro con los extranjeros; éstos no son más que hombres, es decir, nada a sus ojos. Este inconveniente es inevitable, pero débil. Lo esencial es ser bueno con las personas con las que uno vive» (L'Emile, I, págs. 248-), siempre se podrá contraponer el no menos citado, 
ejemplarizados por antonomasia en la utilización del mismo término latino para el extranjero y el enemigo -hostes - y en la noción griega de bárbaro.

Y es que no cabe olvidar que el propio proceso de constitución del Estado moderno como Estado nacional está presidido por el objetivo de homogeneidad social (cultural, lingüística, religiosa, pero no económica, no social en el sentido de igualdad, de fin de los estamentos o de las clases: lo primero no llegará hasta la revolución francesa y lo segundo todavía es para rato). En efecto, como ha mostrado Gellner, la civilización industrial precisó para su constitución y desarrollo una cultura desarrollada, homogénea y favorecedora de la movilidad social y económica, que requiere una cierta independencia política, un Estado propio. Eso sólo era posible en el marco de una estructura política que asegurase dichas condiciones: el Estado nacional. En efecto, es ese Estado el que exige la homogeneidad jurídico-política (el monopolio de la violencia legítima, del derecho), pero no menos requiere, impone la homogeneidad cultural, y eso significa en primer lugar, religiosa y lingüística y étnica: por eso el principio cuius regis eius religio y por eso también la desaparición de las identidades plurales como precio de esa construcción. Eso es lo que muestran también los trabajos de Burke $^{14}$, en línea con las tesis de Gellner, que es el nacimiento del Estado moderno lo que marca la ilusión de una cultura propia, «pura», al poner fin al pluralismo cultural de la Baja Edad Media. Es ese Estado el que impone coactivamente una unanimidad religiosa, moral y cultural mediante la unificación jurídica que hace posible el sometimiento universal a un poder central y la erradicación de toda pauta o conducta que se oponga al proceso de unificación (racionalización patológica) que alcanza también a la cultura cotidiana para que haya sólo un espacio de comunicación comunitaria, persiguiendo toda diferencia, toda herejía (propia elección). Ese proceso, anticipado en no pocos de sus elementos en la construcción de España como Estado moderno, bajo la hegemonía de Castilla y Aragón (que luego será la de Castilla), se ha repetido históricamente en la construcción de los imperios multinacionales (tanto en el caso del imperio austro-húngaro como en el más reciente, el proyecto de un Estado socialista que superase el problema de las nacionalidades, que no otro es el objetivo de la U.R.S.S. levantada por Stalin y el de la Yugoslavia de Tito). En realidad, según una opinión que comparto, lo que podemos aprender de

«Tengo por máxima indiscutible: quienquiera que no haya visto más que a un pueblo, en vez de conocer a los hombres, no conoce más que a las personas con las que ha vivido» (L'Emile, V, pág. 827).

${ }^{14}$ Cfr. Burke, 1991. 
la historia es que el principal factor de exclusión que conocemos (doy por descontado, y no pienso discutirlo, que las formas autoritarias, dictatoriales, totalitarias de comunidad política son el mayor factor de exclusión; lo que interesa ahora es otra cosa), no lo constituye tanto el Estado, ni aún el Estado nacional, sino sobre todo el Estado-nacional liberal, o, lo que es más claro, quien lo dirige: una clase social que se identifica por tales adjetivos, o, si se prefiere, los grupos no necesariamente nacionales de poder económico -social, cultural, informativo, político- que controlan las decisiones que adoptan las instituciones que marcan el orden de las cosas. Eso es lo que puede hacer del Estado -a través del mercado- una «máquina de exclusión».

Pues bien, mientras nos ponemos de acuerdo acerca de si la solución definitiva es la democracia mundial o una organización universal de las naciones, tareas en cualquier caso destinadas a muy futuras generaciones, me parece muy difícil negar que el mejor instrumento con el que contamos para avanzar es el artefacto que ha tratado de reducir al máximo esa exclusión, el Estado con vocación más universalizante, el que ha tratado de identificarse al máximo con todos los miembros de la sociedad que constituyen de hecho la comunidad política, es decir, lo que conocemos como Estado social (aunque algunos hablen de categorías «reales», como Estado asistencial o Estado del bienestar), la modalidad de Estado de Derecho que ha tratado de romper con la abstracción característica de esa figura, tal y como la concibieran los «individualistas posesivos» que la teorizaron, según sabemos desde MacPherson. En efecto, en el universalismo abstracto de ese primer modelo, el Estado liberal de Derecho, la real extensión de los derechos teóricamente atribuidos a todos no se produce, porque el sujeto es restringido, no universal: los sujetos incluidos son los que responden realmente al retrato del homo iuridicus (el individuo, ciudadano, propietario, varón, blanco), el único que es libre e igual el único que actúa -participa, es incluido- en los dos foros que le constituyen como tal, la asamblea y el mercado; frente a ello, las modalidades de exclusión (las derivadas de la ausencia de riqueza -los trabajadores por cuenta ajena-, del género -la mujer, básicamente a través de la institucionalización de los espacios de lo público y lo privado-, del parangón de normalidad, etc.) abarcan a la mayoría de la población. Por el contrario, la forma o estadio que denominamos social, trata de garantizar efectivamente tal extensión de los derechos para todos. El problema hoy, como sabemos, es que la tantas veces anunciada crisis de este modelo (recuérdese la tesis de O'Connor), se ha producido de tal forma que la única alternativa posible parece la del fin de la historia: de nuevo el modelo liberal, el minimal State que renuncia a los intentos 
de universalización en buena parte de los denominados «derechos sociales» (pensiones, sanidad, educación); y si eso es así para con sus ciudadanos, ¿qué no sucederá con los que «vienen de fuera»? ¡Malos tiempos para los extranjeros (extra comunitarios, obviamente), para los inmigrantes «económicos»! Claro está que no se trata de ignorar su problema, sino de establecer medidas de control, a la vista de la rentabilidad de su presencia en términos de legitimidad que se obtiene por el recurso a la necesidad del orden público y la seguridad (con la consiguiente aceptación de medidas que se traducen inevitablemente en debilitamiento de las garantías, así como de las restricciones económicas), todo ello cubierto por la coartada paternalista de «proteger a los extranjeros de los brotes de racismo y xenofobia que surgen como consecuencia de la situación de 'barca llena'».

Quizá la manifestación más clara de lo que trato de recordar sea la aparición (el refuerzo, según las versiones) de lo que Balibar ${ }^{15}$ llama «fronteras interiores», junto al incremento de lo que, en la versión del mismo Balibar o de Castel $^{16}$, podríamos calificar como el relanzamiento de la condición de vulnerabilidad o, con Dahrendorf ${ }^{17}$, la aparición de una franja social («subclase del ghetto» la llaman otros), que incluye a parados permanentes, pobres estancados en su condición de miseria, grupos étnicos desaventajados, etc (nuestro «Cuarto Mundo») que, por así decirlo, «han caído a través de las mallas de la red»; son un mero agregado social, una masa (Balibar) más que una clase. Lo que les une es su condición de víctimas, «el hecho de que no se pueden ayudar por sí mismos (salvo en cuanto individuos)... Necesitan catalizadores y agentes externos o un cambio de valores». Lo significativo es que se trata de no-sujetos, de seres que no cuentan, de los que la sociedad puede prescindir (al menos, tratar de apartar o ignorar) porque no los necesita. Quizá es aún más significativo, como escribe Walzer ${ }^{18}$ nuestro desasosiego o incomodidad ante su existencia, que se traduce, por ejemplo, en el hecho de que ni siquiera sabemos cómo llamarlos: los desposeídos, los perdedores,

${ }^{15}$ Cfr. su Les frontiéres de la democratie, así como Race, Nation, classe. Les identités ambigües (con I Wallerstein), París, La Decouverte, 1988. Una exposición tan clara como breve en «Inegalités, fractionnement social, exclusion» (en VV.AA., Affichard-De Foucault, cds.), Justice social et inegalités, París, Esprit, 1992.

${ }^{16} \mathrm{Cfr}$. sus «De la exclusion comme état á la vulnerabilité comme processus» y «De l'indigence á l'exclusion: la dessaffiliation», en VV.AA., Face á l'exclusion, París, Esprit, 1991. Asimismo, «De l'exclusion á la vulnerabilité», en (en VV.AA., Affichard-De Foucault, eds.), Justice social et inegalités, París, Esprit, 1992.

${ }^{17}$ Dahrendorf, R., «Cittadinanza: una nuova agenda per il cambiamento», Sociologia del diritto, 1/1993, págs. 6 ss. Dahrendorf sigue en buena medida el análisis de W. J. Wilson. págs. 100-101.

${ }^{18} \mathrm{Cfr}$, Walzer, M., «L'esclusione, l'ingiustizia e lo stato democratico», Micromega, 1992, 
los «nuevos pobres» ${ }^{19}$. Se agudiza así la contradicción intima del concepto de ciudadanía, por cuanto es perfectamente posible que coexista la atribución de derechos a cuantos son ciudadanos con una situación de dualización como la que ejemplifica, por poner un caso visible, el incremento de los homeless en los EE.UU. Por supuesto, todo ello sin contar con el grado de exclusión que deriva de la condición de no ciudadano, y no sólo por la difícil justificación de la diferencia de títulos para el reconocimiento de derechos entre ciudadanos y extranjeros, sino incluso dentro del propio Estado, si, como consecuencia de los flujos migratorios, se produce lo que los estudiosos ${ }^{20}$-como Pastore siguiendo los trabajos de Hammar, y también los de Bauböck, Balbo, Castles, Leca, Costa-Lascoux o Wihtol der Wenden- llaman zonas intermedias, dando lugar cada vez más a situaciones de penumbra desde el punto de vista de la certeza en la definición del status jurídico, y a los conflictos propios de la convivencia entre sujetos no estrictamente homologables, que se refleja, por ejemplo, en la tensión entre universalismo y reivindicación de los propios códigos de identidad. Así, se desdibujan las fronteras entre los ciudadanos y los extranjeros, esa categoría de cierre social, esa dicotomía perfecta, funcional para el supuesto del Estado-nacional, y cabe distinguir hasta cinco tipos de situaciones entre los extremos de ciudadano y extranjero, cuyo prototipo es el extranjero denized.

$\mathrm{Y}$ es que si, como recuerda Walzer, la existencia social consiste en poder tomar parte en la distribución de bienes, la ciudadanía, o, mejor, la condición de pertenencia al grupo (pues Walzer distingue

${ }^{19}$ Ballesteros, J., «Los derechos de los nuevos pobres», en VV.AA., (ed. J. Ballesteros), Derechos humanos, Madrid, Tecnos, 1992.

${ }^{20}$ Cfr. Pastore, M. «I 'Cittadini inmigrati: differenze di status e incertezza dei diritti», VV.AA., Contributi in tema di pluralismo giuridico (Papers presentati al Seminario sul pluralismo giuridico. Universitá di Torino, 30.X.91), Materiali di discussione e di Ricerca, Q. 3/1992. De Hammar puede consultarse, además del colectivo editado bajo su dirección, European Inmigration Policy: A comparative Study, Cambridge, C. U. P, 1985, su Democracy and the nation state: Aliens, denizens and citizens in a world of international migration, Aldershot, Avesbury, 1992. Cfr. asimismo, Bauböck, R., «Migration and Citizenship», New Community, 18-1, 1991; Balbo, L., «Cittadini, cittadini-dimezatti, non-cittadini», Inchiesta, XX, 90 (1990); Castles, S., «The guest-worker in Western Europe: an Obituary», International Migration Review, XX-4; Leca, J «Nazionalitá e cittadinanza nell'Europa delle immigrazioni», en VV.AA., Italia, Europa e nuove immigrazioni, Torino, Ed. Fondaz. Agnelli, 1990; Costa Lascoux, J., De l'inmigré au citoyen, París, La Documentation Française 1989; Wihtol der Wenden, C., Les inmigrés et la politique, París, Presses de la Fondation Nationale des Sciences Politiques, 1988, y Wihtol der Wenden, C., «The absence of rights: the Position of illegal Immigrants», en Layton-Henry, Z. (ed), The political rights of immigrant workers, London, Sage, 1992. 
claramente entre una y otra), se revela como el bien primario. El problema se plantea en dos órdenes:

a) De un lado, la «confusión» entre pertenencia (digamos nacionalidad) y ciudadanía, que permite establecer una exclusión institucional, la de los extranjeros. Y aquí, la solución del pretendido universalismo puesto en pie por el formalismo jurídico propio del liberalismo, hace agua por momentos. La solución, en realidad, consistía en negar el problema o ignorarlo: no hay exclusión porque por definición los extranjeros no pueden entrar en el reparto. De paso, convengamos en que los tradicionales oponentes de ese modelo, los comunitaristas (nadie se acuerda ya de los marxistas), tampoco han aportado mucha claridad: en resumidas cuentas, con el cierre en el grupo social o comunidad (nacional, étnica, lingüística, cultural) los «otros» siguen perdiendo.

b) De otra parte, encontramos otra fuente para la exclusión: la que, en el interior del grupo, padecen de hecho quienes, perteneciendo a él, son apartados de la distribución de bienes por la hegemonía de alguno de los principios sucesivamente elegidos para tal adjudicación: el mercado, el mérito, la necesidad. Esa tendencia se refuerza hoy con las reclamaciones de la recuperación del protagonismo de la sociedad civil, de la espontaneidad social cuyo paradigma sería (?!) el mercado. Pero, como muestra a las claras, por ejemplo, el análisis de esa «subclase de ghetto» de la que habla Dahrendorf, está claro que el criterio de eficacia y competencia (y la «generosa» y «positiva» hipótesis de la igual autonomía individual) no sirven, por ejemplo, para que grupos sociales semejantes se sitúen siquiera en el umbral que les permita las primeras oportunidades vitales. Y la pregunta es si una exclusión que, de hecho o institucionalmente, abarca a toda una categoría de sujetos, puede dejar de afectar a la estabilidad social. Una versión de la ciudadanía que no sea, al menos tendencialmente, un proyecto universal, no puede dejar de constituir, para emplear los términos del propio Dahrendorf, «una penosa cobertura del privilegio».

El ciclo de desarrollo económico vivido entre los años 1945-1970 parece irrepetible: la creencia en el pleno empleo (en la condición coyuntural del paro) ha sido abandonada. Hoy, como ha dejado escrito en alguna ocasión G. Peces-Barba, de nuevo la malthusiana parábola del banquete parece convertirse en regla de oro, signo del señorío de la ratio economica sobre el Derecho, la Ética, la Política. La biblia de la ortodoxia económica impone en la actualidad (basta echar un vistazo a las recetas del FMI o del Banco Mundial) el aumento de la financiación de la economía y no la socialización de la riqueza. El coste de paro, de incremento de vulnerabilidad que 
implican para sectores crecientes de la población esas directrices monetaristas puede ser afrontado (no sin enormes dificultades) en países en los que las clases obreras tienen mecanismos de resistencia y solidaridad; en los demás (y ese parece ser nuestro caso) no sucede así: la consecuencia es que aumentará la exclusión, y el modo de empezar es por los extranjeros, pero no se detiene ahí. Ha comenzado un proceso de vulnerabilidad cuyo primer elemento es la precarización del trabajo y las políticas que tienen como resultado el cierre de los cauces que permitían lo que Hoggart ${ }^{21}$ denominara la porosidad progresiva entre las dos clases antagónicas, los mecanismos transversales que permitían un puente: la propiedad de la vivienda, la asistencia contra los riesgos sociales (enfermedad, vejez, desempleo), el acceso a la educación, etc. El aumento del desempleo afecta no sólo a los jóvenes sino a un porcentaje de adultos para los que la recuperación del empleo se torna casi imposible. Todo eso significa, como ha advertido por ejemplo Gaullier, la desestructuración de los ciclos de vida social, configurados en gran medida por la sucesión de las etapas laborales y que conducen a la gestión del tiempo de ocio y de retirada de la vida activa también como factores de estabilidad social; eso es tanto como decir que las consecuencias no se reducen al ámbito laboral, que alcanzan no sólo a la integración social que el trabajo produce, sino a la estabilidad social misma, a la integración social en un orden en el que estén suficientemente garantizados las necesidades y derechos básicos. Se crea así -la expresión es de Castel- una especie de «no-man's land social «que, además, comienza a heredarse; se potencia un proceso de vulnerabilidad que amenaza con extender de forma insoportable los estados de exclusión». Como advierte Walzer ${ }^{22}$, son ellos quienes «participan sólo en una mínima parte en el bienestar de sus países, soportan el peso de la crisis económica, son expulsados de las mejores escuelas y de los mejores puestos, llevan por todas partes los signos de los perdedores. Así reproducimos las exclusiones internas del mundo antiguo: los sin-derechos, los sin-poder, los parados, los marginados». Que se sostenga frente a ello el mito de la exclusión justa (justificada al menos como inevitable, como exigida por las reglas de la «racionalidad económica», contradice la noción misma de justicia.

Precisamente es lo que se advierte en el nuevo tipo de conflicto para el que resulta funcional el mensaje de emergencia social que hoy se liga como una amenaza derivada de la inmigración: son concretamente

${ }^{21}$ Hoggart, R., La cultura de la pobreza (cito por la trad. francesa La culture du pauvre, Ed. de Minuit, París, 1970).

${ }^{22}$ Walzer, M., «L'esclusione, l’ingiustizia e lo stato democratico», Micromega, 1992, pág. 100. 
los sectores más desfavorecidos de la población los que experimentan como un privilegio tanto su condición de ciudadanos como la de trabajadores. Ya no se trata de una situación de derecho, y por ello el enfrentamiento con quienes pueden privarnos de ella es brutal: vale todo (es decir, las respuestas de violencia propias del racismo, por ejemplo). La coartada es, además, que tal presencia rompe o al menos es una amenaza respecto al mínimo de homogeneidad social imprescindible (no ya de la homogeneidad racial o cultural, sino de la posibilidad misma de que todos los nacionales puedan disfrutar de un mínimo de condiciones materiales: puesto de trabajo estable, prestaciones sanitarias, educativas, asistenciales, etc.). Junto a ello, además, se aduce la puesta en cuestión del «patriotismo constitucional», en la medida en que -se dice- no comparten los valores y principios propios de nuestro ordenamiento jurídico constítucional (y es que, mal que pese a Habermas, resulta muy difícil disociar la dimensión étnico-cultural y la jurídica por lo que se refiere a las reglas de juego básicas). En el fondo de este planteamiento late el eco del mensaje rousoniano que exige desterrar del Estado a quien no acate la religión civil y que se manifiesta, asimismo, en la inscripción de los galeotes añorada también por Rousseau en el Contrato social: «obligar a ser libres». La homogeneidad impuesta no es el mejor terreno para la democracia que es un régimen con voluntad de inclusión. Vuelvo a Dahrendorf: «la auténtica verificación de la fuerza de los derechos de ciudadanía es la heterogeneidad. El respeto común a los títulos de acceso a los bienes fundamentales, atribuidos a personas diferentes por su origen, cultura o credo pone a prueba la combinación de identidad y variedad que es el núcleo de la sociedad civil civilizada... por eso... la ciudadanía no será nunca completa mientras no exista una ciudadanía mundial» ${ }^{23}$. La idea de exclusión es incompatible con esa noción de la ciudadanía, con su condición de status del que quedarían excluidos por definición categorías sociales enteras. Frente a estas manifestaciones de exclusión, resulta difícil imaginar las razones que pueden permitir la preferencia por la «espontaneidad social», «el libre juego del mercado» y por tanto, el desmantelamiento de las únicas garantías frente a ello, las que ofrece no la gracia o la buena voluntad de quienes puedan sentirse generosos, sino la vinculación que establece el Derecho, y eso, al menos, es tanto como reconocer la necesidad (mientras no aparezca en el horizonte un sustituto seguro) de la propuesta que conocemos por Estado social. Es lo que advierte Walzer: «Ante la existencia de grupos excluidos, la justicia exige un esfuerzo público para permitir a los miembros de esos grupos volver a entrar en la sociedad

\footnotetext{
${ }^{23}$ Dahrendorf, art. cit., 15.
} 
y actuar de forma independiente en todas las esferas distributivas. Es precisamente el fin de dos bienes sociales estrechamente vinculados: welfare y educación». Se trata en definitiva de un deber social que no puede confiarse sólo a los voluntarios sociales (que son «agencias de inclusión»), porque los sistemas asistenciales mismos dependen de formas organizadas e institucionales de asistencia. El Estado debe facilitar el trabajo de las asociaciones surgidas de la sociedad civil, pero no sólo eso, pues la mejor forma de luchar contra la exclusión puede ser aumentar la cantidad de bienes disponibles, más que redistribuir lo que ya existe y ello es fruto de decisiones políticas. Por eso puede concluir, con una tesis elemental que atraviesa, según creo, buena parte del trabajo de Elías Díaz, no sólo como profesor o investigador, sino como intelectual, esto es, que el Estado no puede ser neutral o no comprometerse con el significado de los bienes sociales elementales, pues «lo que caracteriza una comunidad política democrática es el reconocimiento de que todas aquellas transacciones sociales que llevan a los ciudadanos a los márgenes, que producen una clase de excluidos son... siempre injustas» ${ }^{24}$. En otras palabras, y por volver a la provocación (al debate), eso significa que el Estado no puede abandonar la exigencia de solidaridad como una virtud social, sino, que debe considerarla, al contrario, como un principio exigible, como parte del contenido de la justicia, al igual que lo son la igualdad y la libertad.

${ }^{24}$ Walzer, art. cit., pág. 113. 
